\title{
Lidil
}

Revue de linguistique et de didactique des langues

$57 \mid 2018$

Démarches créatives, détours artistiques et appropriation des langues

\section{Le projet artistique : une puissance maïeutique pour la compétence langagière}

Art As a Powerful Maieutics of Linguistic Skills

Maud Sérusclat-Natale et Maryse Adam-Maillet

\section{(2) OpenEdition}

Journals

Édition électronique

URL : http://journals.openedition.org/lidil/4908

DOI : $10.4000 /$ lidil.4908

ISSN : 1960-6052

Éditeur

UGA Éditions/Université Grenoble Alpes

Édition imprimée

ISBN : 978-2-37747-048-8

ISSN : $1146-6480$

Référence électronique

Maud Sérusclat-Natale et Maryse Adam-Maillet, « Le projet artistique : une puissance maïeutique pour la compétence langagière », Lidil [En ligne], 57 | 2018, mis en ligne le 01 mai 2018, consulté le 19 avril 2019. URL : http://journals.openedition.org/lidil/4908; DOI : 10.4000/lidil.4908

Ce document a été généré automatiquement le 19 avril 2019

(C) Lidil 


\title{
Le projet artistique : une puissance maïeutique pour la compétence langagière
}

\author{
Art As a Powerful Maieutics of Linguistic Skills
}

Maud Sérusclat-Natale et Maryse Adam-Maillet

\section{Introduction}

1 Le projet artistique que nous allons aborder se déroule dans une unité pédagogique pour élèves allophones arrivants (UPE2A) accueillant des élèves de plus de 16 ans. Ces unités ont pris la suite des classes fermées (CLA ou CLAD classe d'accueil ou d'adaptation). Leur fonctionnement inclusif est décrit dans les textes officiels de l'Éducation nationale depuis $2012^{1}$. Les UPE2A lycée sont récentes et rares en France. Implantées en fonction des besoins des territoires et des politiques éducatives locales, elles ont pour mission de dispenser un «enseignement de langue française comme discipline et comme langue instrumentale des autres disciplines ${ }^{2}$ " qui s'organise en fonction des besoins linguistiques et scolaires de chaque élève. Elles doivent installer toutes les stratégies de compensation et d'étayage permettant à l'élève allophone arrivant (EAA) de pouvoir suivre progressivement les cours de la classe ordinaire dans laquelle il est inscrit et d'y réussir jusqu'au diplôme. La démarche didactique s'y individualise entièrement. Les enseignants de français langue étrangère - français langue de seconde ou langue de scolarisation (FLE-FLS) - ne sont pas soumis à des programmes, mais doivent permettre à l'élève d'entrer simultanément dans le français oral et écrit, dans le français de scolarisation et de s'adapter aux exigences relatives aux curricula de chaque section d'inclusion. Un tel défi érige les UPE2A en lieux d'expérimentation pédagogique constants. Si des travaux de recherche en didactique du FLE-FLS existent (Davin-Chnane, 2006 ; Cherqui \& Peutot, 2015) aucun n'interroge sur le fond les modalités d'enseignement pour les EAA de plus de 16 ans. L'expérience du projet artistique que nous développerons innove dans ce contexte relativement inexploré. Par ailleurs, si les bénéfices liés aux 
pratiques artistiques en termes d'acculturation scolaire ou d'appropriation de la langue cible ont déjà été démontrés (Pierra, 2006 ; Clerc, Corier, Longeac \& Oustric, 2007 ; Auger, 2010), ils ne portent pas sur les dispositifs UPE2A lycée actuels et n'abordent pas la question de la collaboration interinstitutionnelle consistant à faire entrer physiquement dans les classes des artistes, ni la dynamique et la méthodologie d'une co-construction. Les concepteurs de ce projet, la scène nationale de l'agglomération de Montbéliard (désormais MA) et le Centre académique pour la scolarisation des enfants allophones nouvellement arrivés et des enfants de familles itinérantes et de voyageurs (désormais $\left(\mathrm{ASNAV}^{3}\right)$ de l'académie de Besançon, partagent une mission de service public d'éducation artistique et culturelle ${ }^{4}$, ainsi que la volonté de produire toutes les médiations nécessaires pour que les publics dits «éloignés » dans le vocabulaire des institutions impliquées, accèdent à la création contemporaine. Cette volonté commune, s'agissant des EAA, s'appuie, non sur les représentations d'un public scolaire démuni mais, au contraire, sur l'hypothèse de compétences interculturelles et plurilingues de haut niveau (Abdallah-Pretceille, 1999) développées dans un cadre de mobilité ou migration (Moro, 2010) et sur l'hypothèse du projet artistique comme booster de l'apprentissage de la langue française cible (Auger, Terrades \& Talagrand, 2007 ; Aden, 2009 ; Armand, 2013).

\section{2. « Le bruissement des langues »}

\subsection{Contexte de création}

2 Le « bruissement des langues » fait partie d'un projet plus large, intitulé Parlemonde, mené conjointement par MA et le CASNAV durant l'année scolaire 2016-2017. Six artistes européens ont collaboré avec une centaine d'EAA âgés de 10 à 19 ans et leurs enseignants. Parlemonde n'a pas été conçu comme projet de recherche. Sa finalité première est esthétique, et non académique, mais sa méthodologie s'appuie sur les travaux en didactique des langues. Les résidences ont eu lieu dans trois UPE2A, une classe de CM2 et un dispositif d'hébergement pour mineurs non accompagnés dépendant de l'aide sociale à l'enfance, au sein de l'aire urbaine de Belfort Montbéliard, riche d'une histoire migratoire séculaire et de langues variées. Au principe de Parlemonde: la volonté de lier geste artistique contemporain et didactique récente des langues et des cultures. Ainsi les artistes plurilingues extérieurs aux institutions éducatives ont travaillé aux côtés des enseignants des UPE2A et des éducateurs de jeunes en situation de migration ${ }^{5}$, avec la médiation du CASNAV. Une telle configuration visait à favoriser la confrontation à l'altérité (Spaëth, 2014) et à potentialiser l'engagement des divers acteurs. Elle orientait vers les finalités politiques souhaitées dès le départ pour le projet: exposer, de façon valorisante et sécurisée, le travail commun des artistes et des jeunes dans l'espace public de Montbéliard, comme n'importe quelle autre création assurée par MA. En termes logistiques, c'est le plus ample ${ }^{6}$ projet jamais piloté par la plateforme créative de la structure. Parlemonde a exposé six créations d'élèves et d'artistes lors d'un festival au centre historique de Montbéliard les 4 et 5 mai 2017. L'ensemble du projet (biographies, notes d'intention et productions des participants) est communiqué sur le site compagnon ${ }^{7}$ qui éclairera cet article. On s'arrêtera plus particulièrement sur «le bruissement des langues » (désormais LBDL), travail de l'artiste plasticien, poète et performer Frédéric Dumond, mené avec les EAA de l'UPE2A du lycée Follereau de Belfort. 


\subsection{Ancrage théorique et hypothèses}

Par sa complexité plurimodale, LBDL dépasse les relations d'apprentissage réservées dans l'institution scolaire à l'espace ritualisé et fermé de la classe de langue (Cicurel, 2011). Il s'agit, pour les acteurs du projet, de faire émerger collectivement de nouvelles stratégies pour renforcer par l'expérience commune de la créativité, l'envie et le plaisir d'apprendre, tout en ciblant l'enseignement-apprentissage d'une langue particulièrement exigeante dans ses variétés de production écrite : le français de scolarisation (VerdelhanBourgade, 2002; Klein, 2012). L'enjeu est de s'appuyer sur les langues premières des participants ${ }^{8}$, enfants comme adultes, de mobiliser dans les interactions et les productions l'ensemble de leur répertoire linguistique (Coste, Moore \& Zarate, 1997, 2009; Moore, 2006) grâce aux activités de création et de favoriser la communication translangagière (Canagarajah, 2011 ; García \& Wei, 2014) dans le groupe multilingue que constitue l'UPE2A, en explorant comment translanguaging et créativité se renforceraient l'un l'autre (Aden, 2009; Armand, 2013 ; García \& Wei, 2014). Comment les liens entre les individus se densifient-ils, comment leurs apprentissages ou créations s'en trouvent-ils stimulés, accélérés, cependant que se déconstruisent pour tous les représentations premières (Jodelet, 1991) liées à l'altérité? Nous pensions également qu'une telle expérience intensive de co-apprentissage linguistique et d'éducation interculturelle pourrait constituer une situation d'enseignement enrichissante pour les élèves et de formation modélisable pour les adultes.

\subsection{Corpus et méthodologie}

Les œuvres co-créées sont :

Tableau 1. - Euvres produites.

\begin{tabular}{|l|c|}
\hline Euvres & Formes de l'œuvre \\
\hline Alphabet & Affiche (Taille : 310X150) \\
\hline De la où je suis né(e) & Poèmes plurilingues affiche (Taille : 310X150) \\
\hline Les bouches & Installation visuelle et sonore \\
& 3 écrans LED de $2 \mathrm{~m}$ avec 3 sources de diffusion séparées \\
& $\begin{array}{c}\text { Superposition des bouches et superposition des sons. Pas de } \\
\text { synchronisation de l'image entre les } 3 \text { sources. } \\
\text { Format 1920X1080 }\end{array}$ \\
\hline Pièce sonore 1 & Installation 6 enceintes dans l'escalier \\
\hline Pièce sonore 2 & Table d'écoute avec casques \\
\hline Totem reprenant l'alphabet & Construction métallique : épaisseur 3mm, hauteur 2003mm, poids \\
inventé & $115 \mathrm{~kg}$ \\
\hline
\end{tabular}

5 Ayant observé et/ou participé aux séances d'atelier ainsi qu'aux divers moments de conception, création et production du projet, nous avons recueilli et joint au corpus les éléments suivants : 
Tableau 2. - Corpus.

\begin{tabular}{|l|l|}
\hline Traces & Codages \\
\hline Note d'intention & NI \\
\hline Fiche technique des installation & FT \\
\hline Un entretien Frédéric Dumond et Maud Natale & EA \\
\hline 2 vidéos de Frédéric Dumond présentant son travail à la structure & VA1 et VA2 \\
\hline $\begin{array}{l}5 \text { enregistrements de réunions de travail et de mise en cuvre dans la } \\
\text { structure }\end{array}$ & RT1 à RT5 \\
\hline 8 entretiens Maud Natale avec élèves & EE1 à EE8 \\
\hline 20 questionnaires écrits des élèves & Q1 à Q20 \\
\hline Un entretien post projet Maud Natale avec Yannick Marzin, directeur de & ED \\
\hline MA entretien Maud Natale avec responsable plateforme créative de MA & EPT1 \\
\hline Un entretien Maud Natale avec chargée de projet plateforme créative de MA & EPT2 \\
\hline Un poème de sortie de résidence de Frédéric Dumond & P \\
\hline 41 productions écrites (hors œuvres) des participants & PEP1 à PEP41 \\
\hline
\end{tabular}

On s'appuiera sur les entretiens semi-directifs des enseignants et personnels concernés, ainsi que de l'artiste et des élèves impliqués, mais aussi sur l'observation simple et/ou participante des séances d'atelier, sur les enregistrements des réunions participatives avec l'équipe artistique, ainsi que sur l'exploitation des productions écrites en L1-L2, des procédures de traduction et de mise en valeur artistique. Enfin, nous nous sommes également fondées sur notre formation littéraire, qui envisage la poésie et plus généralement l'art, comme une des voies majeures de la connaissance.

\section{Du détour à la reconnaissance}

\subsection{La langue au cœur du travail de l'artiste}

7 Dans un tel contexte, le fait que l'œuvre de l'artiste questionne la langue ou les enjeux de la rencontre entre les langues et les cultures est primordial. Frédéric Dumond déploie, par l'écriture, la vidéo, le son, la poésie, l'installation et la performance une même œuvre : Glossolalie, un poème constellation écrit dans les 7000 langues du monde et dont l'un des enjeux oulipien réside dans l'impossible achèvement. Il explore la phénoménologie des langues et de leur contact, et à travers elles, la manifestation de soi et d'autrui. Lors d'une réunion d'équipe (RT2), l'artiste propose cette définition de la langue :

C'est le médium puissant et majeur qui tout à la fois permet de se représenter le monde, de s'en faire une idée et en même temps le met à distance. Les mots et leur grammaire sont ce qui permet de comprendre l'autre, l'autre que soi et en même temps le soi dans l'autre. La langue est outil de l'entendement, mais tout autant masque, puisqu'elle est aussi traduction, transcription en concepts de l'expérience. La langue donne accès au monde $\mathrm{ET}$ nous en distrait.

8 Une telle approche évoque le propos poétique de Novarina (1999) et reste sensorielle au premier chef :

Le langage est anthropogène : il apparait de l'homme en parlant. La langue est la source de notre chair, notre danse d'apparition. L'écriture est l'exercice d'un muscle spirituel: la pensée va se trouver par le ralenti [...] Un déroulement traversal, transversal, un respirement par où on va toucher jusque dans la terre des mots. (p. 62)

Le principe de faire vivre aux élèves le protocole de travail de l'artiste, d'expérimenter par un temps d'imprégnation «chaque mot comme chaque souffle, comme chaque 
fragment du monde » comme une "affaire de perception et d'expérience » a été adopté. Le titre de l'œuvre, "le bruissement des langues ", nait avec l'idée de l'explorationexposition artistique de la rencontre des langues des participants. L'artiste a produit pour MA une note d'intention, qui n'a pas fait l'objet d'une analyse avec les élèves, car on a privilégié une entrée pratique avant toute méta-explicitation.

Image 1. - Note d'intention.

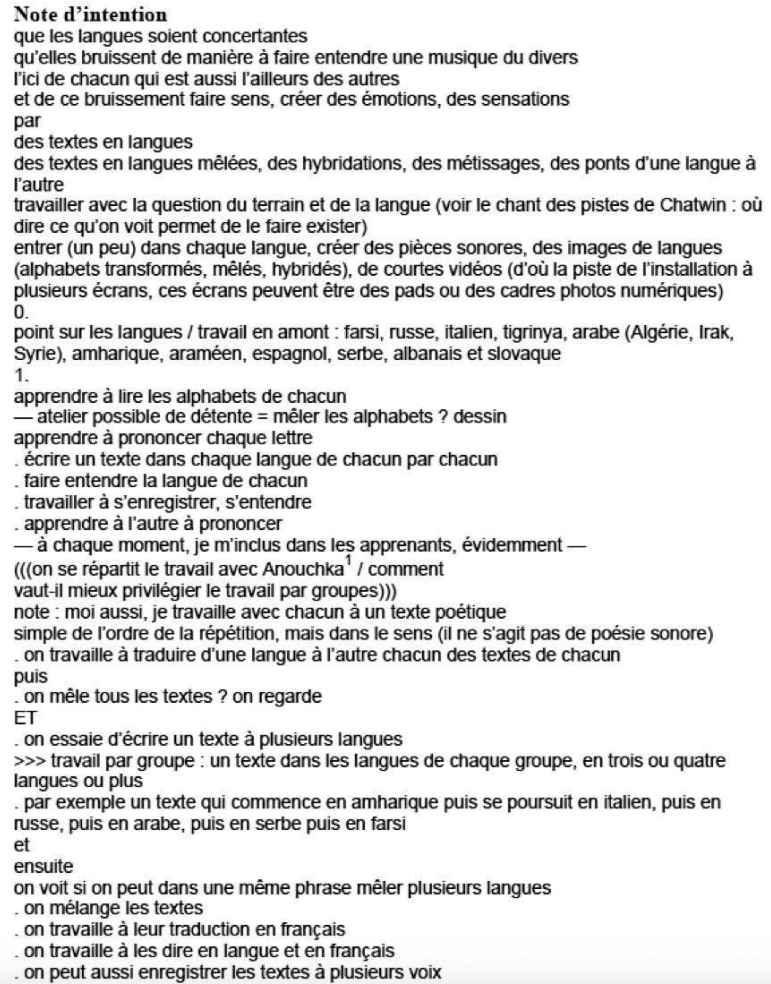

La forme de cette expression originale marque quatre éléments essentiels. D'abord la sensorialité de la langue, mobilisant le corps du poète et de l'apprenant, mais aussi leur psychisme profond (Fönagy, 1983). L'espace de jeu ouvert par l'expérimentation sensorielle de la langue de l'autre, de sa propre langue et de la langue cible, élabore l'UPE2A comme un espace translangagier de communication et d'interaction, espace dont l'école se prive la plupart du temps en se focalisant sur l'écrit monolingue. L'apprentissage du FLS ne saurait pourtant se passer de l'exploration des L1 et plus largement du répertoire linguistique, à faire émerger en tant que socle de création artistique au rebours des représentations communes bloquantes (Adam-Maillet, 2012; Auger \& Cadet, 2016).

11 Le second point réside dans l'absence de directivité de la note. Le processus de création collective fonde l'action sans la pré-contraindre. LBDL déploie un espace de coopération horizontale entre tous les acteurs, sans projeter de représentation à priori sur les capacités à agir des élèves ni sur leurs désirs. Appuyé sur les langues légitimées des apprenants, l'artiste ne programme pas un monolithe, mais une œuvre plurielle, fragmentée; il mise sur la richesse des interactions produites, sur la variété matérielle possible des supports (visuels, sonores, textuels) mis à disposition techniquement par la structure et le lycée. 
12 La dimension poétique intrinsèque de la note d'intention rappelle aussi Roland Barthes (1984) dans le Bruissement de la langue : « C'est le frisson du sens que j'interroge en écoutant le bruissement du langage ». Figurant le respirement (Novarina, 1999), notamment à travers les effets de mise en page, d'alinéa et une ponctuation forte, Frédéric Dumond affirme la littérarité (Jakobson, 1973) de l'expérience. La co-création avec des EAA, porteurs de compétences en L1 et en français diverses, n'affecte pas la portée ou l'exigence littéraires de la résidence. L'axiologie de LBDL se rapproche des propos de Judet de la Combe et Wiesmann (2006) :

[...] Pour le passage d'une langue naturelle à une autre, le problème n'est pas simplement technique; encore faut-il qu'il y ait de l'intérêt et du respect pour ces langues, de l'une à l'autre, et donc qu'une opération de reconnaissance ait lieu. [...] En tant que langue, [une langue minoritaire] est ouverte à toutes les autres et aux différents usages possibles d'une langue. Si elle doit retrouver une place dans l'espace public de la communication et de l'éducation, dont l'État a en charge l'organisation et la liberté, elle devra faire valoir ses droits au nom d'une idée universelle de la langue et de la communication. Ce sont chaque fois des opérations de « décentrement » qui sont requises, de sortie hors d'un usage constitué et limité, non pas au nom d'une idée englobante de la culture, comme identité donnée, ou de l'interculturalité, comme mise en commun des cultures ou comme pur échange entre des entités culturelles constituées, mais, plus simplement, au nom de la nécessité qu'un espace commun assure la possibilité que soit authentiquement reconnue la valeur des différentes activités langagières des individus. (p. 111)

Enfin, en contractualisant l'horizontalité des relations de travail et la coopération, l'artiste se place comme apprenant et non comme enseignant. Il ne s'agit pas de transformer un résultat créateur en support prétexte à des activités langagières décontextualisées, mais d'alimenter le foyer des démarches artistiques, personnelles ou interinstitutionnelles en instaurant "un pré-discours comportemental qui inscrit les êtres dans la relation » (Cyrulnik, cité par Schmidt, 2006, p. 98).

\subsection{La prise de risque : un catalyseur}

L'accueil d'un artiste en résidence, dépossédant l'enseignant de toute posture magistrale et instaurant une telle horizontalité, s'est vécu au lycée comme une prise de risque que nous avions largement sous-estimée dans nos projections de départ. En effet, même si elle échappe aux contraintes habituelles des programmes, l'UPE2A doit inclure les EAA dans l'ensemble des filières et sections du lycée, dans lesquelles ils sont soumis au même rythme d'apprentissage que les autres élèves. Ils préparent des examens (CAP, bac divers) et leur implication dans un projet artistique sur plusieurs jours consécutifs réclame des médiations tendues. La coordonnatrice de l'UPE2A a choisi de susciter et non d'imposer la participation de chacun. Mais, comment décrire à l'avance les activités alors qu'elles jailliront précisément en contexte de création ? Elle a présenté LBDL à tous les EAA et a dédié au projet la totalité des heures du dispositif sur six jours, soit 42 heures d'intervention de l'artiste. Puis sont venues les négociations pour le rattrapage des cours, des évaluations, etc. Une fois réglée la communication au sein d'un lycée comptant près de 200 enseignants et 2000 élèves, les réserves émises par les EAA, parents ou professeurs, pouvaient encore inquiéter. Selon eux, les élèves, « déjà fragiles » allaient « perdre » des heures et seraient mis « en danger scolairement ».

Dans son entretien (EA), lorsqu'on lui demande s'il a perçu des spécificités liées au public allophone, Frédéric Dumond répond : 
Je ne vois pas de différence. [...] À part cette histoire d'efficacité plutôt que dans la création, la question de la rentabilité car les filles [...] n'imaginaient pas que cela pourrait leur être utile. Grosse exigence scolaire chez certains, chez d'autres moins. [Un temps] Plus largement: plus d'exigence scolaire qu'avec les autres publics avec qui j'ai travaillé.

L'obsession du rendement provient-elle des stéréotypes des élèves, de leur habitus scolaire, de leur fort mandat de réussite (Goï, 2015), ou de réticences exprimées par les enseignants, insécurisés par ce qu'ils perçoivent comme intrusion dans un espace où ils régulent d'ordinaire le temps et les progressions? Dans notre travail au profit des EAA, nous constatons au quotidien la puissance et la permanence des représentations glottophages (Calvet, 1974) installées dans l'esprit de la plupart des acteurs du système éducatif. Dans ce lycée comme ailleurs, dans les discours oraux et écrits comme dans les pratiques, on exige des EAA un engagement absolu dans le travail. Ils doivent souvent être exemplaires et excellents, et renoncer à l'usage scolaire de leurs langues premières. Les archives du CASNAV attestent à l'envi du caractère répandu de cet état d'esprit. 20 élèves sur 26 se risquent pourtant à coopérer avec une structure culturelle et un artiste.

Tous les acteurs du projet, d'une façon générale, ont pris un risque et la structure la première. Yannick Marzin, son directeur, explique au cours de l'entretien ED :

Prendre un risque à l'endroit où je suis, c'est ouvrir autant de fenêtres que possible. [...] La prise de risque c'est presque le moteur de tout, un petit peu comme le mouvement de Trisha Brown. C'est la prise le risque qui donne le mouvement. [...] Trisha Brown, c'est hyper compliqué à danser, c'est hyper technique, c'est un mouvement qui donne l'impression d'un relâcher constant et infini. Et évidemment, c'est tout sauf cela. Et en même temps, tu le rates ou plutôt tu ne rentres jamais dedans si tu n'arrives pas à te laisser tomber et à te rattraper, parce que la danse c'est ça [...]. [Un temps] À un certain moment, ce qui va moi me motiver, c'est la prise de risque.

Interrogé pour savoir s'il considérait l'allophonie des participants comme un risque supplémentaire, il déclare :

La pratique de la danse m'a habitué à la difficulté d'être compris, ou en tout cas de trouver du sens. Les langues étrangères ne me font jamais peur, de la même manière que j'ai vraiment démarré mon expérience artistique comme danseur et la danse est abstraite : il n'y a pas de narration. Ça ne me fait pas peur, je n'ai aucune appréhension de dire " on va présenter telle pièce en langue étrangère ", au même titre que je programme de la danse contemporaine : c'est demander un effort, une gymnastique d'esprit [...] je n'ai pas peur d'aller vers la difficulté, et le positionnement d'une structure culturelle est très clair pour moi : sur un territoire enclavé on doit justement casser l'enclave. C'est la question du sens qui compte.

Le propos fait écho à celui de Frédéric Dumond qui, dans cet extrait de "C'est-à-dire ${ }^{9}$ " évoque la peur du nouveau sous le terme de la «distance» entre les langues ou de « résistance »: 
Image 2. - Extrait du poème écrit par l'artiste à la fin de sa résidence.

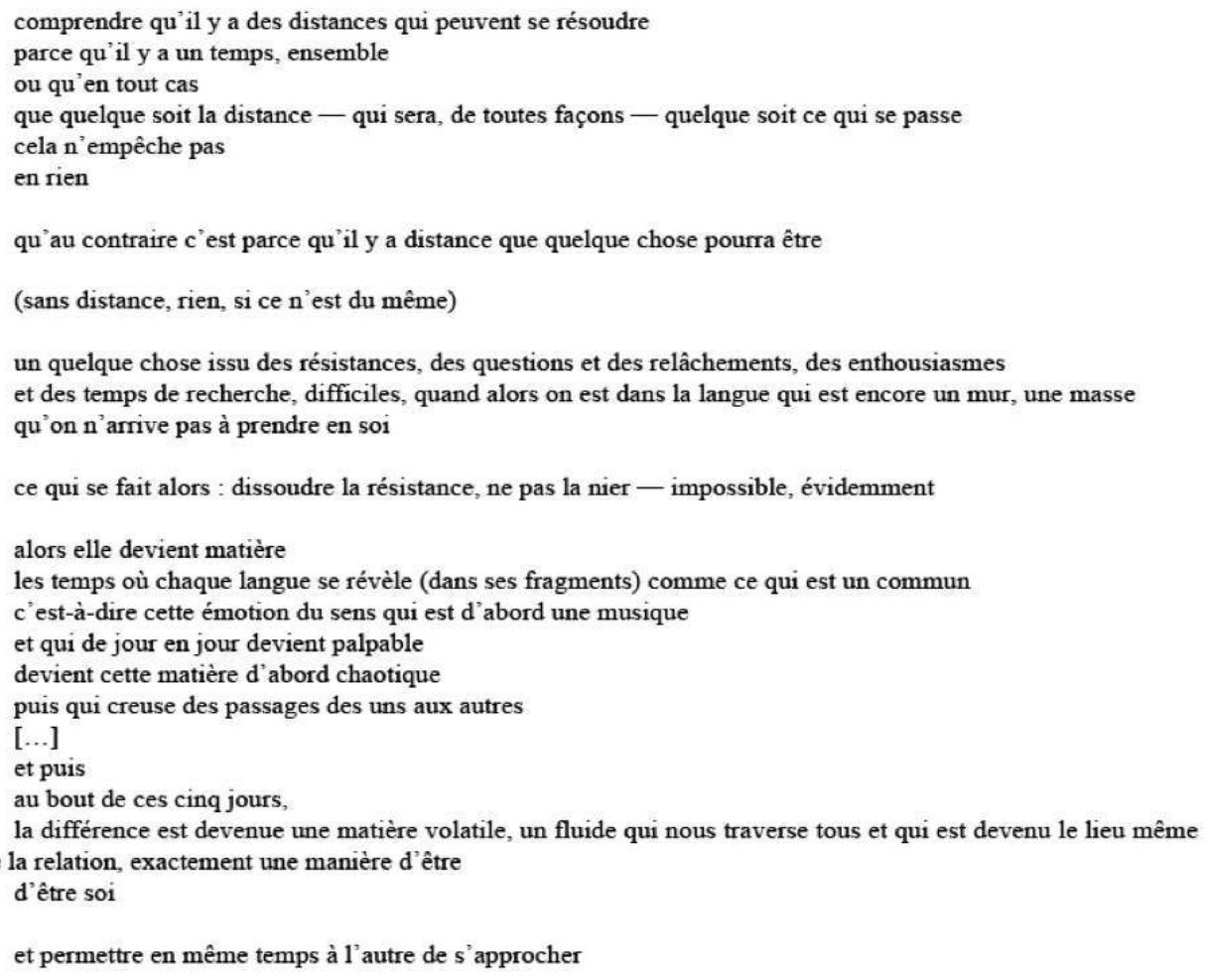

La distance devient la matière d'une création subversive. Lubart (2003) catégorise ainsi les facultés conatives autorisant la créativité : traits de personnalité, styles cognitifs et motivation. Parmi les traits de personnalité entretenant « des relations significatives avec la créativité » seraient « la tolérance à l'ambiguïté » ainsi que « la prise de risque », en jeu lors d'un projet artistique à l'école. Les textes écrits lors de la première journée d'immersion font écho au poids de l'exigence scolaire et aux risques encourus par qui s'y dérobe, comme le montre ce texte (PEP4MD) écrit par une participante :

Image 3. - Production d'une participante.

Chacun d'entre nous, naît dans un abîme, chacun différent que l'autre.

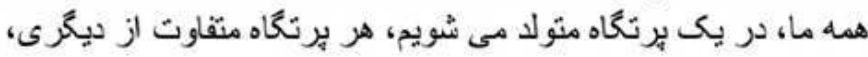

\section{Dans l'abîme de la vie, il y a des problèmes, des problèmes du genre des atomes incassables.

$$
\text { در برنكاه زندگى؛ مشكلات غوطه ور اند، مشكلاتى از جنس اتم هاى شكست نابذير ، }
$$

21 Dans les questionnaires et entretiens des EAA, en réponse à la question "qu'as-tu ressenti ? », aucun élève n'évoque la peur. Certains parlent « d'une grande liberté » ou « d'un sentiment bien et bizarre à la fois ». Ab. explique : «J'étais timide au début. Je suis fier de moi aujourd'hui parce que je suis allé jusqu'au bout. C'est parce que j'ai aimé, c'est parce que c'était risqué aussi. » (EE8AB)

Dans la mesure où aucun élève n'avait participé auparavant à un projet artistique, ni n'avait manifesté de connaissances théoriques sur l'art, on peut penser que les réticences 
évoquées avant la réalisation de LBDL signifient que les jeunes avaient intériorisé les représentations et discours doxiques sur l'inutilité de l'art.

\subsection{Du détour à l'engagement}

Dans les entretiens et questionnaires, les élèves mentionnent que c'est au moment du projet que l'espace de l'UPE2A devient un lieu à part, protégé des fonctionnements ordinaires, à l'écart. Ces propos nous ont invitées à repenser la question du détour. Nous pensions que le projet artistique lui-même représenterait un détour aux yeux d'une majorité des acteurs, comme souvent dans les recherches en sciences de l'éducation qui envisagent la pédagogie du détour en direction des publics posant problème à l'institution scolaire comme un subterfuge (Henri-Panabière, Renard \& Thin, 2016), qui contournerait «l'obstacle cognitif» ou le «blocage» de l'élève pour le conduire indirectement vers les savoirs scolaires, y compris par mobilisation de compétences artistiques ou culturelles (Meirieu, 2004). En fait, cette conception s'appuie sur une représentation de l'enseignant comme capable de mobiliser le geste artistique en l'absence de l'artiste lui-même. Or, si un enseignant peut développer une pédagogie créative, elle ne sera pas artistique à moins qu'il ne soit lui-même artiste, ce qui reste une situation exceptionnelle.

D'autre part, les théoriciens des sciences de l'éducation postulent implicitement que le geste artistique ne serait pas une fin en soi, mais l'instrument ou le moyen d'atteindre des objectifs de rentabilité scolaire quantifiables. Or, les expériences et les conceptions philosophiques plaçant l'art au premier plan, partagées par tous les partenaires impliqués, ne nous permettaient pas d'adhérer à la représentation commune du projet comme détour. Nous voulions montrer qu'il était plutôt catalyseur. De surcroit, le projet artistique conduit à une reconnaissance publique et globale des compétences plurielles des EAA, touchant directement, émotionnellement, les enseignants, enfants, parents, spectateurs, loin des visions verticales ou ségrégatives. Nous l'envisageons ici comme clé de voute de l'empowerment (Calvès, 2009) des EAA à leurs propres yeux et aux yeux des autres, pairs et adultes. Acteur et sujet de son parcours au sein d'un collectif : l'EAA s'engage plutôt qu'il est engagé, grâce à la mise en place d'activités plurilingues médiées par l'artiste, l'enseignant et les apprenants entre eux. Selon les termes de Marie-Rose Moro $^{10}$ :

C'est le processus social, culturel, identitaire, dans lequel sont [les EAA] qui est vulnérabilité mais il peut devenir créativité au sens où on peut faire quelque chose avec ces différences à partir du moment où on les reconnait, on les inscrit, on les valorise, où elles ne restent pas hors cadre, hors école.

En tant qu'engagement commun, le projet artistique permet de renverser l'empêchement supposé de la «barrière de la langue » en rendant à l'EAA, dans un mouvement physique et symbolique, son corps et sa voix. Par conséquent, de la même façon que la création artistique n'est pas un résultat mais un mouvement, un process, la prise de conscience par les élèves de leurs compétences langagières relève d'une dynamique et d'une dialectique. En l'état actuel des curricula, le projet artistique est l'endroit le plus propice pour initier cette dynamique, dont on espère qu'elle continuera à produire ses effets dans les cours ordinaires. 


\subsection{Vivre ses langues comme " espace potentiel ${ }^{11}$ »}

Image 4. - Pièce « Les bouches ».

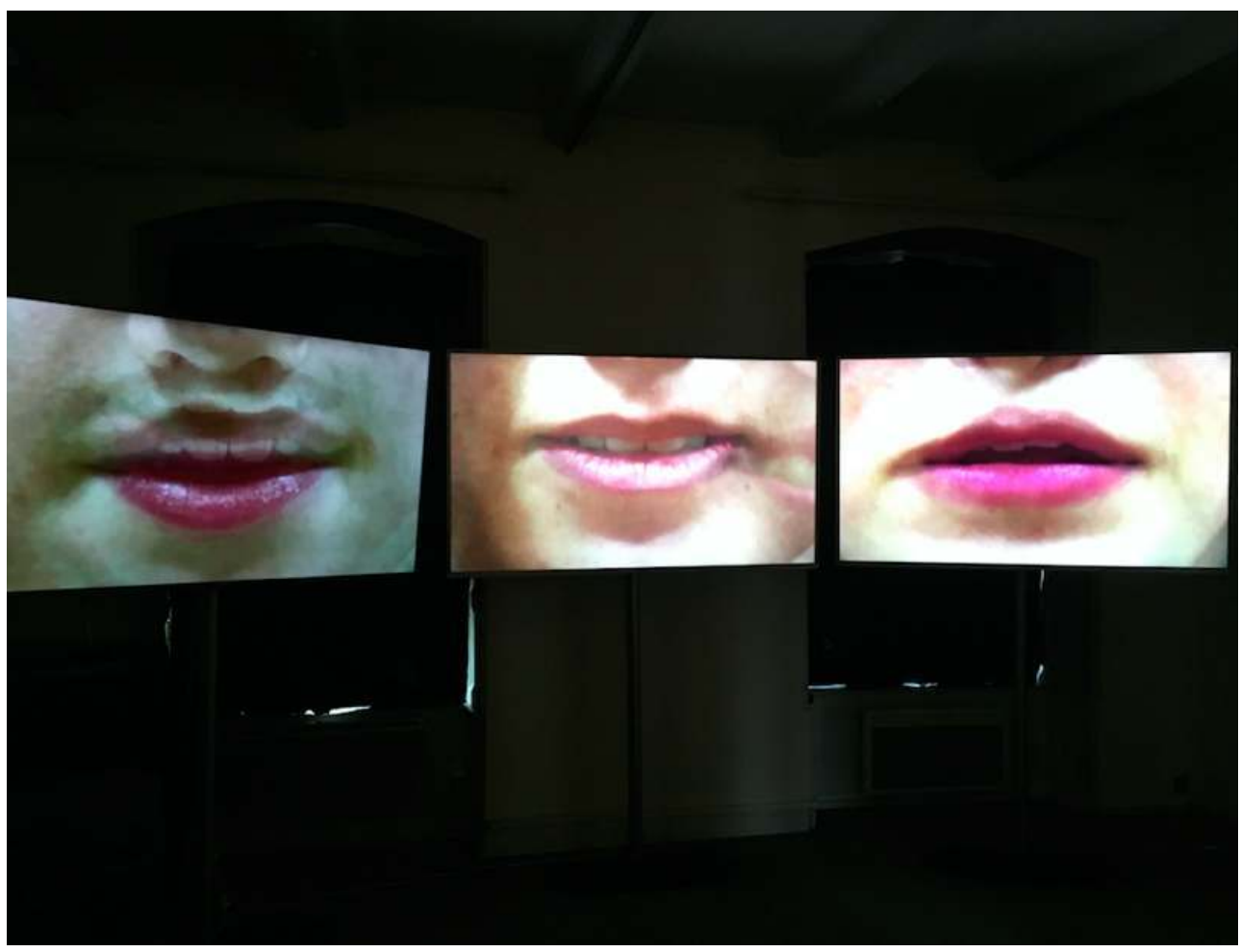

Le principe d'horizontalité place chacun en posture d'apprenant : la création procède par allers retours entre langues et personnes. Un sens de groupe nait du "partage du vécu commun» (Aguilar-Río, 2013 p. 140, rappelant Dörnyei, 2007) propre à une dynamique relationnelle et créative. LBDL correspond au premier moment où les élèves de l'UPE2A inclus dans des classes ordinaires différentes ont pu, selon eux, «faire groupe » en tant que membres d'une "vraie classe ». Ils soulignent dans les entretiens l'importance du sentiment de sécurité qui découle de ce «faire groupe » valorisant les différences et insistent sur le contraste avec la classe ordinaire :

J'ai rencontré [à l'UPE2A] des gens qui avaient les mêmes problèmes que moi, j'ai pu oser parler. (Q1AC)

J'ai aimé qu'on travaille en groupe, surtout quand on travaillait en langue française. Je suis stressée en classe, il y a des jours je pleure à l'école parce que je me sens trop en difficulté, pas là. Parce que je suis comme les autres, je suis étrangère ici, comme tout le monde. (Q2ME)

J'ai appris à communiquer avec les autres, on est comme une petite famille, on se soutient les uns les autres, j'ai aimé travailler ainsi. Ici je sais que c'est pas important de savoir si je vais faire des fautes ou pas, je me lance et je dis ce que j'ai à dire. (EE1I)

Ces propos rappellent d'autres recherches (Armand, Lory \& Rousseau, 2013) qui ont montré que les apprenants mobilisés par de telles pratiques collectives ressentent le climat scolaire comme "moins menaçant», renforcent leur sentiment d'efficacité personnelle et leur motivation (Puozzo, 2013), ce qui a des répercussions positives sur les apprentissages langagiers. Certains participants comme A. ${ }^{12}$, vont plus loin et associent le «faire groupe » aux interactions produites dans la dynamique de création: «J'ai parlé 
tout le temps avec les autres élèves, les profs, dans l'actuation ${ }^{13}$.» (Q20A) Le groupe constitué permet une prise de parole sécurisée, gratuite, ludique parce que LBDL mobilise tout le répertoire des élèves conscientisant ainsi leur compétence plurilingue (Coste, Moore \& Zarate, 2009). Chaque activité de production est proposée à l'EAA dans la langue de son choix. Un élève sur quatre dans les entretiens utilise le mot « soulagement » quant à cette autorisation. La valorisation des langues premières s'effectue non seulement en légitimant leur usage au sein de l'espace de travail mais en le transformant en condition nécessaire à l'accomplissement, non pas de la tâche mais de l'acte de création. On est loin de ce que rapporte $\mathrm{M}$. lorsqu'on lui demande si et comment elle utilise ses langues à l'école :

Ma langue à l'école, d'habitude je la parle doucement, pour ne pas déranger. Là [pendant le projet] j'ai parlé et écrit en arabe puis traduit. Là, j'ai découvert que l'arabe pouvait me servir pour apprendre le français. (EE3M)

An., de son côté, a souligné que son plurilinguisme était une chance :

En utilisant mes langues, j'ai appris que je suis forte car je parle plein de langues, j'étais fière de moi-même. (Q8AN)

La légitimation, primordiale, du répertoire linguistique a permis de construire les stratégies individuelles pour le mobiliser. Par exemple, J., observé lors d'une séance, est resté longtemps sans écrire le premier jour parce qu'il «ne se voyait pas le faire en français ${ }^{14} »$. Interrogé sur son répertoire linguistique il déclare :

Je parle l'araméen, j'ai étudié l'arabe et l'anglais à l'école... [Un temps] je connais le kurde aussi, je le parle, le lis et l'écris. [Un temps] Et le syriaque aussi. Mais ça ne sert à rien tout ça. (Q9J)

J. pense que ses langues sont trop éloignées du français et ne sait pas quelle stratégie utiliser pour appréhender ce qui les rapproche plutôt que ce qui les éloigne du français cible. Il n'est pas encore entré dans la production écrite. C'est participant au projet, par la médiation de l'adulte, qu'il découvre comment s'appuyer sur son répertoire pour passer d'une langue à l'autre. C'est également à cette occasion qu'il le dévoile complètement et parvient à décrire ses compétences dans chaque langue. On dépasse alors la liste des langues déclarées ou reconnues par les élèves. Le répertoire linguistique devient pour les participants et les adultes « un ensemble dynamique des variétés linguistiques maitrisées par un même locuteur, à quelque degré et pour quelque usage que ce soit » (Aden, 2012, p. 269). LBDL redéfinit l'UPE2A aux yeux de tous: au-delà du seul enseignement du français scolaire, elle devient un lieu où chacun apprend à partir des compétences déjà là, un lieu qui rappelle que « l'apprentissage d'une nouvelle langue ne se fait pas en rupture avec la ou les langues déjà parlées, mais au contraire sur la base d'une expansion dynamique des compétences langagières » (Aden, 2012, p. 269).

Enfin, la valorisation de la compétence plurilingue des EAA a permis l'émergence d'une compétence essentielle et invisibilisée dans un système éducatif monolingue, la compétence de médiation, définie par Aden (2012) comme étant :

[...] à la fois le produit (le sens émergent), le moyen (catalyseur) et le résultat (la réussite de l'interaction) et prend des formes variées, [...] multimodales selon les interlangues des sujets en interaction. (p. 270)

La mobiliser invite à croiser les répertoires langagiers dans un contexte d'apprentissage nouveau, celui de la création. LBDL constitue cet espace potentialisant, en tant que manifestation dynamique développant motivation, coopération, confiance, entrée dans les apprentissages langagiers, y compris en français (Auger, Terrades \& Talagrand, 2007). 
Au-delà, son enjeu est la création collective d'une œuvre plurielle sur « l'entre langues » ou «l'avant langue ${ }^{15}$ exposée publiquement, figurant et poétisant une dynamique du lien entre les langues.

Image 5. - Syllabaire Parlemonde.

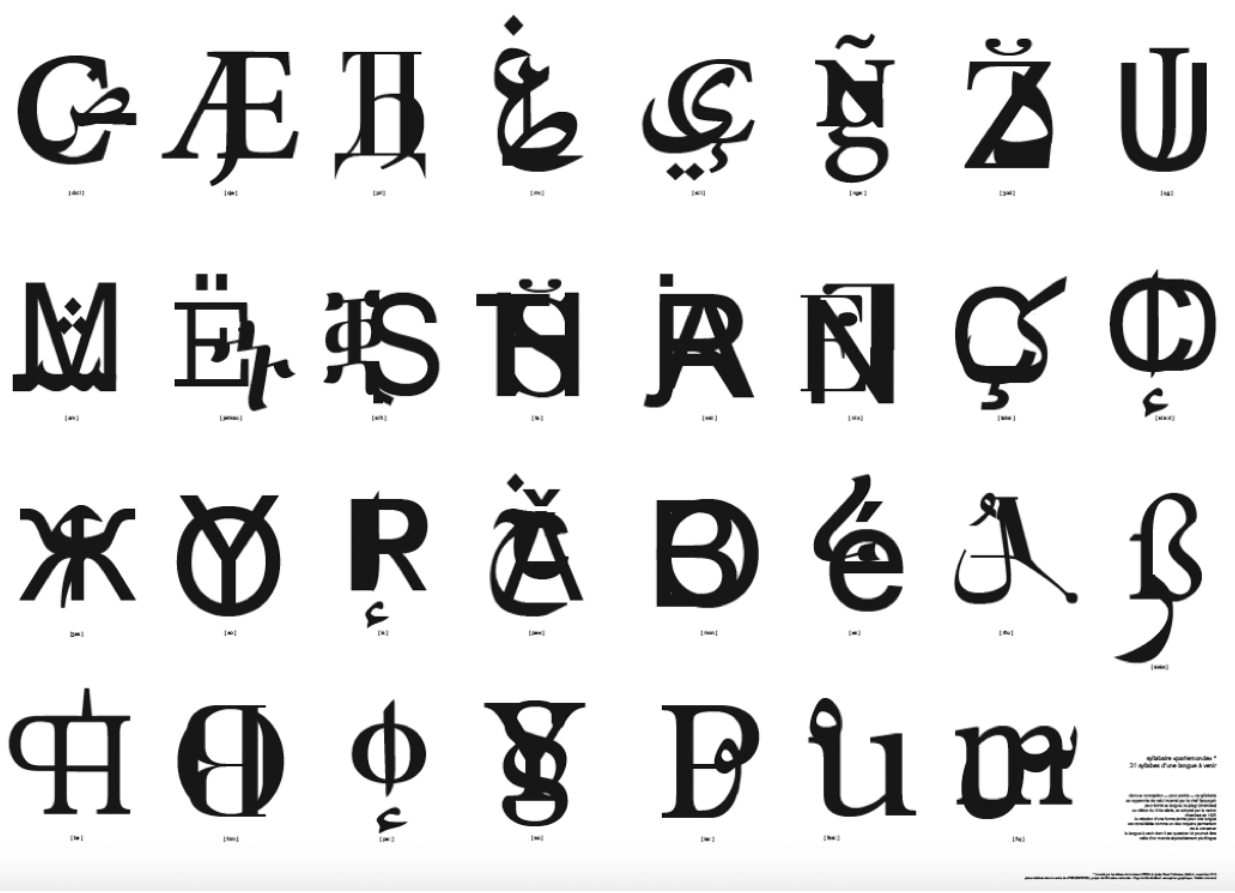

\subsection{S'exposer c'est créer du sens}

Finaliser ensemble une œuvre concrète à visée esthétique importe (Auger, 2010), mais la montrer et participer à sa réception en dévoile aussi les process, puisque les participants assurent la médiation avec le public. Le moment de l'exposition, évoqué ainsi par Evans "quand celui-qui-voit surgit pour examiner notre œuvre ${ }^{16}$ ", transcende les frontières entre école et Cité. Yannick Marzin le souligne ${ }^{17}$ :

[La forme festivalière] permet non seulement de rassembler les acteurs du projet, leurs familles et le public, mais surtout de ne pas tomber dans l'anecdotique d'une simple sortie d'atelier.

Le public confère sa légitimité au projet, il ne s'agit plus d'un détour. Novarina (2012) l'explique ainsi dans la Quatrième personne du singulier :

Tous - chacun libre - délivrent le livre, par leurs émotions, par leur rire, leur respiration. Si cette opération vraie n'a pas lieu, le spectacle reste prisonnier de luimême.

L'une des chargée de projets de MA constate :

Ils sont impressionnants ces jeunes. Ils avaient envie d'être entendus, dans leurs langues maternelles et en français, on a bien vu l'importance des deux versants, et le lien avec le public, la volonté d'être bien compris par le public. (EPT1)

Les élèves ont réalisé que leur travail serait exposé un mois avant le festival, lors d'un temps de résidence à $\mathrm{MA}$, consacré à la scénographie des présentations des œuvres produites avec Parlemonde. Être dans le lieu, écrire les feuilles de salle avec l'équipe des relations publiques, être formé à l'accueil, participer à la vie de la structure et voir son 
travail être exposé donc légitimé, au même titre que toute autre œuvre artistique, a été le déclencheur d'une autre prise de conscience. LBDL avait produit de l'art: les textes, l'alphabet, le Totem allaient être lus, vécus, reçus par d'autres. Les deux types de motivations impliquées dans la créativité que décrit Lubart (2003), intrinsèque et extrinsèque, fusionnent. Une élève comprend, que c'est pour cela qu'elle n'aura "pas de note ", mais que son travail sera récompensé différemment puisqu'il sera vu. Une autre prend également conscience de la valeur du travail accompli : «Je ne m'étais pas rendue compte qu'on avait fait “ça”." Lubart (2003, rééd. 2015) évoquant les travaux de Mcclelland (1962) parle d'ailleurs d'un sentiment d'accomplissement lié «au désir de réussir une tâche en rapport avec le travail effectué, et [...] par la reconnaissance sociale externe que l'individu assouvit le besoin de constater sa réussite » (p. 140).

Dans leurs entretiens et questionnaires, les jeunes expriment l'étonnement devant la beauté de l'exposition:

C'était vraiment trop bien fait, ça m'a étonnée, j'étais fière. (Q7I)

L'installation du travail était magnifique. J'étais surprise et satisfaite car je ne pensais pas que ça allait donner ça, du coup j'étais plutôt fière. (Q2AN)

J'étais très content et j'étais très fier de notre travail. (Q6J)

C'était la première fois que je voyais quelque chose comme ça. (Q5EH)

J'étais fier de nous et de tous [sic] ce qu'on a fait. (EE2AM)

J'étais fière pour le travail qu'on avait fait. (Q4C)

J'ai aimé parce que c'était pas seulement dans la classe où on apprend des choses, mais aussi avec des expériences comme ce projet. [...] J'étais fière de ce qu'on a fait. (Q19S)

\section{Conclusion}

LBDL a construit un espace de parole sécurisé et horizontal rappelant la nature contextuelle des connaissances; il a reconnu et légitimé les langues de tous et l'ensemble de leurs usages, y compris leur fonction purement poétique, en premier lieu aux yeux des participants eux-mêmes. C'est le potentiel épistémologique, esthétique, axiologique de la diversité linguistique qui a été valorisé. La déségrégation des EAA au lycée a été renforcée par l'implication de professeurs extérieurs à l'UPE2A: la filière chaudronnerie s'est emparée avec enthousiasme de LBDL en façonnant le Totem. 
Image 6. - Totem.

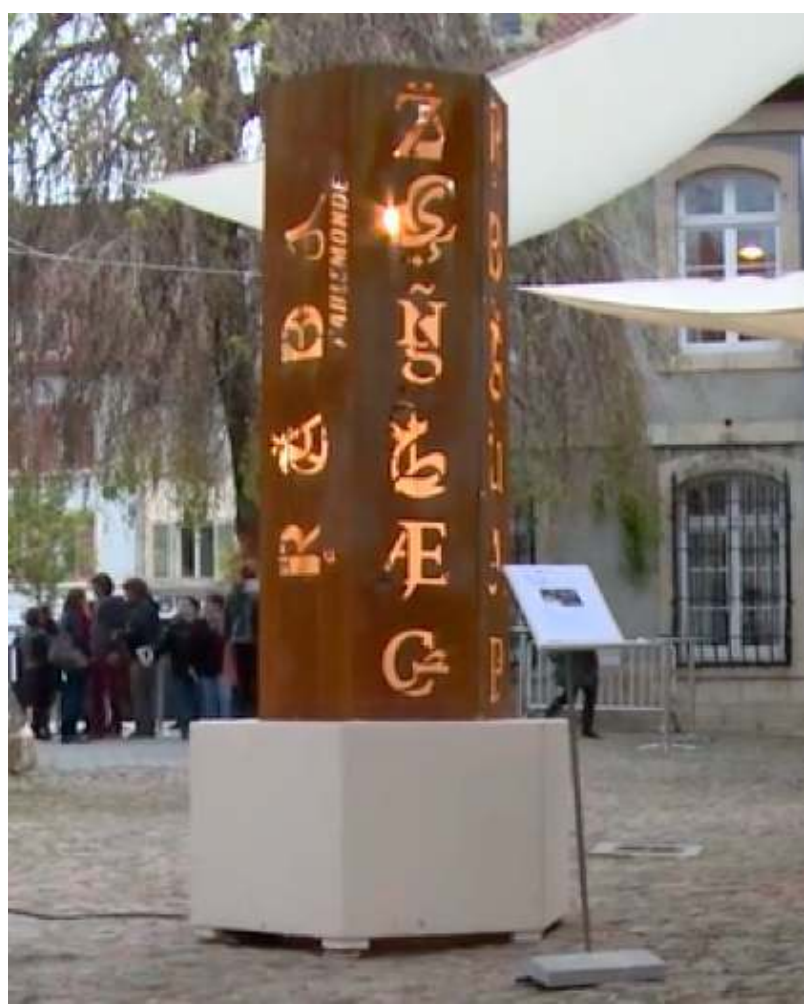

Si les EAA relient peu participation artistique et "métier d'élève » dans leurs questionnaires, ils soulignent presque tous les progrès linguistiques accomplis et les rapportent explicitement au projet. Enfin, ils expriment unanimement leur désir de renouveler une telle expérience.

Du côté de MA, Parlemonde a creusé la conscience de l'importance des questions plurilingues dans le champ des politiques artistiques et culturelles :

C'est une expérience qui va nous faire passer à autre chose [...]. Artistiquement cela a été fécond, cela a produit du sens [...] il ne faut pas qu'on s'arrête là. Ça aussi, ça ne me l'avait jamais fait avec les autres projets, cette envie de poursuivre. (Extrait de EPT1)

41 Yannick Marzin conclut, après le festival Parlemonde :

Avec Parlemonde on a touché un niveau de réalisation et de réflexion qu'on n'avait jamais atteint jusqu'à présent. Ce qui m'interroge c'est que je ne l'ai pas vu venir [... ] Je le sais pour les artistes, ça les a bougés. Parlemonde [devient] un cycle [qui] apparait comme une évidence, un numéro deux à l'horizon, et fait passer la plateforme dans une autre phase, qu'elle ne soit pas simplement un objet de production et de diffusion mais qu'elle soit un objet [...] qui nourrit le sens. [...] C'est notre rôle de travailler ces questions de reconnaissance sociale, et ça devrait être constant. On ne peut pas ne pas poursuivre, c'est du sens qu'on a créé. (ED)

Véritable voie/voix de recours, suscitant l'émergence d'une dynamique du lien, le projet artistique, parce qu'il multiplie les médiations avec une concentration sans équivalent, opère une puissante maïeutique de la compétence langagière. En tant qu'« opération vraie » le projet artistique permet à tous d'être « agis » ensemble par une nouvelle forme de langage, selon la formule de la jeune $\mathrm{S}$. : « [...] cette langue d'art qui contient toutes les langues auteurs du monde. » (Q19S) 
intention de modéliser ce type d'actions, demande particulièrement actuelle des institutions, notamment culturelles, nécessite de mener une recherche qui interroge plus qu'un seul fragment de projet. LBDL et plus généralement Parlemonde ont semblé, pour tous les partenaires, esquisser les contours possibles d'une autre Histoire, collective, fraternelle, articulée avec justesse aux histoires individuelles de chacun (Ricœur, 2004 ; Spaëth, 2014). Afin de répondre à cette demande, le corpus global de Parlemonde constitue aujourd'hui l'objet de la recherche doctorale de l'une des co-auteures et certaines œuvres produites sont devenues des outils de formation pour les chargés de mission du CASNAV. Par ailleurs, d'autres projets artistiques réunissant artistes et EAA sont nés de cette expérience et sont en cours. Parlemonde devient un évènement institué en biennale par MA, la démarche sera renouvelée en 2018-2019. Il ne s'agit donc plus d'une expérience unique qui n'obéirait qu'à la temporalité d'un événement, mais d'une dynamique de fonctionnement partenarial et de coopération transférable dont les invariants seront explorés.

\section{BIBLIOGRAPHIE}

AbDallah-PRetceille, Martine. (1999). L'Éducation interculturelle. Paris, PUF.

ADAM-MAILlet, Maryse. (2012). Les représentations stéréotypées du français : paradoxalement des leviers pour l'apprentissage/enseignement du français comme langue de scolarisation. Dans C. Klein (dir.), Le français comme langue de scolarisation : accompagner, enseigner, évaluer, se former. Paris : Scérén/CNDP-CRDP.

ADEN, Joëlle. (2009). La créativité artistique à l'école : refonder l'acte d'apprendre. Synergie Europe, $4,173-180$.

ADEN, Joëlle. (2012). La médiation linguistique au fondement du sens partagé : vers un paradigme de l'enaction en didactique des langues. ELA - Études de linguistique appliquée, 167, 267-284.

AgUiLAR-Río, Jose-Ignacio. (2013). L'enseignement d'une langue comme pratique émotionnelle : caractérisation d'une performance, ébauche d'une compétence. Lidil, 48, 138-156. Disponible en ligne sur <https://journals.openedition.org/lidil/3323>.

ARMAND, Françoise, LORY, Marie-Paule \& RouSSEAU, Cécile. (2013). « Les histoires, ça montre les personnes dedans, les feelings. Pas possible si pas de théâtre. " (Tahina) - Ateliers d'expression théâtrale plurilingue en classe d'accueil. Lidil, 48, 37-55. Disponible en ligne sur <https:// journals.openedition.org/lidil/3311>.

AUGER, Nathalie. (2010). Élèves nouvellement arrivés en France. Paris : Éditions des archives contemporaines.

AUGER, Nathalie \& CADET, Lucie. (2016). Que révèlent les parcours et les pratiques des enseignants en classe de français ? Éléments de réflexions sur les conflits cognitifs et méthodologiques face aux traditions didactiques. Dans A. Pegaz Paquet \& L. Cadet (dir.), Les langues à l'école, la langue de l'école (p. 47-66). Artois Presses Université. 
AUGER Nathalie, TERRADES Olivier \& TALAGRAND, Richard. (2007). Ateliers littérature et arts pour entrer dans le français langue seconde. ELA - Études de linguistique appliquée, 147, 339-348.

BARTHES, Roland. (1984). Le Bruissement de la langue. Essais critiques IV. Paris : Seuil.

CALVÈs, Anne-Emmanuèle (2009). «Empowerment » : généalogie d'un concept clé du discours contemporain sur le développement ». Tiers Monde, 200, 735-749.

CALVET, Louis-Jean. (1974). Linguistique et colonialisme. Petit traité de glottophagie. Paris : Payot.

CANAGARAJAH, Suresh. (2011). Translanguaging in the Classroom: Emerging Issues for Research and Pedagogy. Applied Linguistics Review, 2, 1-28.

CHERQUi, Guy \& PEUTOT, Fabrice. (2015). Inclure : français de scolarisation et élèves allophones. Paris : Hachette.

CicUREL, Francine. (2011). De l'analyse des interactions en classe de langue à l'agir professoral : une recherche entre linguistique interactionnelle, didactique et théories de l'action. Pratiques, 149-150, 41-55. Disponible en ligne sur <http://pratiques.revues.org/1693> (consulté le 22 mai 2017).

Clerc, Stéphanie, CoRTIER, Claude, LONGEAC, Amandine \& Oustric, Gérard. (2007). Place et intérêt des pratiques artistiques, interculturelles dans les dispositifs d'accueil et d'enseignement/ apprentissage pour les élèves allophones. ELA - Études de linguistique appliquée, 147, 317-328.

Coste, Daniel, Moore, Danièle \& Zarate, Geneviève. (1997, 2009). Compétence plurilingue et pluriculturelle (éd. révisée en 2009). Strasbourg : Conseil de l'Europe.

CYRULNIK, Boris. (2000). Les nourritures affectives. Paris : Odile Jacob.

DAVIN-CHNANE, Fatima. (2006). Français langue étrangère, français langue maternelle/première : quelle passerelle pour l'enseignement du FLS au collège ?, Skholê, hors-série 1, 35-41.

Dumond, Frédéric. Glossolalie. Disponible en ligne sur $<\mathrm{http}$ ://fredericdumond.free.fr $>$ (consulté le 28 décembre 2016).

FöNAGY, Ivan. (1983). La vive voix. Essais de pyscho-phonétique. Paris : Payot.

GARCíA, Ofelia \& WEI, Li. (2014). Translanguaging: Language, Bilingualism and Education. Basingstoke : Palgrave Macmillan.

GoÏ, Cécile. (2015). Langues et rencontre interculturelle en éducation : loyautés, conflits, autorisations. Dans D.-L. Simon (dir.), Accueillir l'enfant et ses langues : rencontres pluridisciplinaires sur le terrain de l'école (p. 95-118). Paris : Riveneuve éditions.

HenRI-PANABIÈRe, Gaële, RenARD, Fanny \& Thin, Daniel. (2016). Des détours pour un retour ? Pratiques pédagogiques et socialisatrices en ateliers relais. Revue française de pédagogie, 183, 71-82. Disponible en ligne sur <http://rfp.revues.org/4168> (consulté le 2 janvier 2017).

JAKOBSON, Roman. (1973). Questions de poétiques. Paris : Seuil.

JODELET, Denise. (1991). Les représentations sociales. Paris : PUF.

JUdET DE LA COMBE, Pierre \& WiesmanN, Heinrich. (2004). L'avenir des langues. Repenser les humanités. Paris : Cerf.

KLEIN, Catherine (dir.). (2012). Le français comme langue de scolarisation : accompagner, enseigner, évaluer, se former. Paris : Scérén/CNDP-CRDP.

LubART, Todd. (2003). Psychologie de la créativité (réédition en 2015). Paris : Armand Colin. 
MCCLELLAND, D. C. (1962). On the Psychodynamics of Creative Physicals Scientists. Dans H. E. Gruber, M. Wertheimer \& G. Terrel (dir.), Contemporary Approaches to Creative Thinking (p. 141-147). New York : Atherton Press.

MEIRIEU, Philippe. (2004). Éducation : peut mieux faire ? Le Monde, 13 décembre 2004.

MOoRE, Danièle. (2006). Plurilinguisme à l'école. Paris : Didier.

Moro, Marie-Rose. (2010). Grandir en situation transculturelle. Disponible en ligne sur < www.infomie.net/spip.php?article957> (consulté le 28 décembre 2016).

NovarinA, Valère. (1999). Devant la parole (réédition en 2009). Paris : P.O.L.

NovARINA, Valère. (2012). La quatrième personne du singulier. Paris : P.O.L.

PIERRA, Gisèle. (2006). Le corps, la voix, le texte. Arts du langage en langue étrangère. Paris :

L'Harmattan.

Puozzo, Isabelle. (2013). Pédagogie de la créativité : de l'émotion à l'apprentissage. Éducation et socialisation, 33. Disponible en ligne sur <http://edso.revues.org/174> (consulté le 15 décembre 2016).

RICEUR, Paul. (2004). Parcours de la reconnaissance. Paris : Gallimard.

SchmidT, Prisca. (2006). Le théâtre comme art de l'apprentissage de la langue étrangère. Spirales, 38, 93-109.

SPAËTH, Valérie. (2014). La question de l'autre en didactique des langues. Glottopol, 23, 160-172.

Disponible en ligne sur <http://glottopol.univ-rouen.fr/telecharger/numero_23/

gpl23_08spaeth.pdf>.

VERDELHAN-BOURGADE, Michèle. (2002). Le français de scolarisation : pour une didactique réaliste. Paris, PUF.

WinNicotT, Donald. (1971). Jeu et réalité. L'espace potentiel. Paris : Gallimard.

\section{ANNEXES}

Annexe 1. - Parlemonde 


\begin{tabular}{|c|c|c|c|}
\hline \multicolumn{4}{|c|}{$1 /$ Les créations } \\
\hline Artistes & Projets & Participants & $\begin{array}{l}\text { Personnels éducation } \\
\text { nationale mobilisés }\end{array}$ \\
\hline $\begin{array}{l}\text { Charlotte Lagrange } \\
\text { Assistée de Antoine } \\
\text { Richard (Son) } \\
\text { Arthur Michel } \\
\text { (lumière) }\end{array}$ & $\begin{array}{l}\text { «Sédiments" } \\
\text { Création d'une fiction } \\
\text { radiophonique puis de } \\
\text { son passage au plateau }\end{array}$ & 20 EAA lycée & $\begin{array}{l}1 \text { enseignant } \\
2 \text { Assistants Pédagogiques } \\
\text { (AP) } \\
2 \text { enseignants remplaçants } \\
\text { 1Chargé de mission } \\
\text { CASNAV (CM) } \\
\text { fin de projet: } \\
8 \text { étudiants BTS } \\
\text { audiovisuels / 2 enseignants }\end{array}$ \\
\hline Wil Mathjis & $\begin{array}{l}\text { «Becoming » } \\
\text { Création de } 5 \text { mini } \\
\text { documentaires (écriture } \\
\text { tournage montage }\end{array}$ & 15 EAA Collège & $\begin{array}{l}1 \text { enseignant } \\
1 \mathrm{AP} \\
1 \mathrm{CM} \text { CASNAV }\end{array}$ \\
\hline Sébastien Fayard & $\begin{array}{l}\text { «Dé-formations » } \\
\text { Création d'une } \\
\text { exposition contenant } 20 \\
\text { photographies }\end{array}$ & $\begin{array}{l}21 \text { élèves } \\
\text { plurilingues de CM2 }\end{array}$ & $\begin{array}{l}2 \text { enseignants } \\
1 \text { CM CASNAV }\end{array}$ \\
\hline $\begin{array}{l}\text { Thomas Boichard } \\
\text { "Yoggyone» }\end{array}$ & $\begin{array}{l}\text { Création d'un album et } \\
\text { de } 3 \text { prestations live }\end{array}$ & $10 \mathrm{EAA}$ & $\begin{array}{l}3 \text { éducateurs } \\
\text { 1CM CASNAV }\end{array}$ \\
\hline David Subal & $\begin{array}{l}\text { "Orientation : un } \\
\text { partage des chemins» } \\
\text { Création de } \\
\text { performances, et de } \\
\text { deux expositio }\end{array}$ & $\begin{array}{l}20 \text { EANA du lycée } \\
\text { Raoul Follereau }\end{array}$ & $\begin{array}{l}1 \text { enseignant } \\
1 \mathrm{AP} \\
1 \text { enseignant remplaçant } \\
1 \mathrm{CM} \text { CASNAV }\end{array}$ \\
\hline Frédéric Dumond & $\begin{array}{l}\text { «Le bruissement des } \\
\text { langues» } \\
\text { Création d'une } \\
\text { exposition visuelle et } \\
\text { sonore, et d'une nouvelle } \\
\text { langue. }\end{array}$ & $\begin{array}{l}20 \text { EANA du lycée } \\
\text { Raoul Follereau }\end{array}$ & $\begin{array}{l}1 \text { enseignant } \\
1 \mathrm{AP} \\
1 \mathrm{CM} \text { CASNAV }\end{array}$ \\
\hline $\begin{array}{l}6 \text { artistes et } 2 \\
\text { collaborateurs }\end{array}$ & 6 œuvres & 86 Participants & 28 personnels \\
\hline
\end{tabular}

Implication de la structure MA scène nationale

\begin{tabular}{|l|l|l|l|}
\hline Périodes & $\begin{array}{l}\text { Période de résidences } \\
\text { des } 6 \text { créations }\end{array}$ & $\begin{array}{l}\text { Rencontre } \\
\text { professionnelle du 4 } \\
\text { mai 2017 }\end{array}$ & $\begin{array}{l}\text { Événement 4 et } 5 \text { mai } \\
2017\end{array}$ \\
\hline $\begin{array}{l}\text { Personnels et } \\
\text { intermittants } \\
\text { mobilisés }\end{array}$ & 20 & 8 & 45 \\
\hline
\end{tabular}

\section{Annexe 2. - Langues des participants}

\begin{tabular}{|c|c|c|c|}
\hline Participants & Langue 1 & Autres langues & \begin{tabular}{|l|} 
Classes \\
d'inclusion
\end{tabular} \\
\hline MD & Farsi & Anglais, arabe, français & $1^{\text {ere }}$ Scientifique \\
\hline $\mathrm{KA}$ & Russe & Anglais, français & $\begin{array}{l}\text { Terminale } \\
\text { économique et } \\
\text { sociale }\end{array}$ \\
\hline 1 & Somali & Français & Terminale CAP \\
\hline IME & Arabe & Espagnol, catalan, français & 2de générale \\
\hline AN & Tchèque & Anglais, français & $\begin{array}{l}\text { Terminale } \\
\text { économique et } \\
\text { sociale }\end{array}$ \\
\hline AM & Soninké & Bambara, arabe, français & Terminale CAP \\
\hline $\mathrm{DE}$ & Albanais & Anglais, français & 2de générale \\
\hline AMA & Arabe & Anglais, français & 2de bac pro \\
\hline $\mathrm{AC}$ & Arabe & Anglais, français & 2de générale \\
\hline $\mathrm{DZ}$ & Serbe & Anglais, français & 2de bac pro \\
\hline NA & Arabe & Anglais, français & 1ère technologique \\
\hline $\mathrm{CA}$ & Araméen & Arabe, anglais, français & $1^{\text {ère }}$ technologique \\
\hline s & Arabe & Anglais, français & 2de générale \\
\hline ME & Arabe & Anglais, français & 2de générale \\
\hline A & Espagnol & Anglais, français & $1^{\text {ère }}$ technologique \\
\hline $\mathrm{AB}$ & Arabe & Espagnol, catalan, français & 2de GT \\
\hline $\mathrm{J}$ & Araméen & $\begin{array}{l}\text { Arabe, syriaque, kurde, anglais, } \\
\text { français }\end{array}$ & 1'ire technologique \\
\hline IM & Arabe & Anglais, français & 2de bac pro \\
\hline JA & Arabe & Italien, français, anglais & \begin{tabular}{|l|} 
Terminale \\
technologique
\end{tabular} \\
\hline
\end{tabular}

\begin{tabular}{|l|l|l|l|}
\hline Participants & $\begin{array}{l}\text { Langues } \\
\text { premières }\end{array}$ & Autres langues & $\begin{array}{l}\text { Classes } \\
\text { d'inclusion }\end{array}$ \\
\hline AZ & Kirghize & Russe, anglais, français & $\begin{array}{l}\text { Terminale } \\
\text { économique et } \\
\text { sociale }\end{array}$ \\
\hline DO & Arabe & Italien, anglais, espagnol, français & 2de générale \\
\hline TE & Serbe & Français, anglais, espagnol & 1'ere Scientifique \\
\hline EM & Albanais & Anglais, français & $\begin{array}{l}\text { Terminale } \\
\text { économique et } \\
\text { sociale }\end{array}$ \\
\hline OR & Albanais & Anglais, français & 2de générale \\
\hline ANI & Arabe & Anglais, français & 2de générale \\
\hline EH & Shimaoré & Français, anglais, arabe, espagnol & 2de générale \\
\hline
\end{tabular}
Tableau 2 : Élèves ayant rejoint Parlemonde après l'écriture du « Bruissement des

\section{NOTES}

1. Circulaire $\mathrm{n}^{\circ} 2012-141$ du 2 octobre 2012. 
2. Ibid., § 2.2 .

3. Pôles d'expertise, instances de coopération et de médiation et centres de ressources et de formation.

4. Extrait du projet artistique et culturel de MA: «La plateforme créative dessine et met en œuvre des projets éducatifs et implicatifs visant l'accès et la participation du plus grand nombre à la création artistique et à la vie culturelle. Elle s'appuie sur la présence d'équipes artistiques en son cœur et sur un réseau de partenaires issus de l'Éducation nationale et du monde associatif pour co-construire des projets originaux conçus sur mesure pour le territoire et pour ses habitants. »

5. Dispositif AMNA de Montbéliard (Accueil mineurs non accompagnés).

6. Voir annexe 1.

7. <http://parlemonde.mascenenationale-creative.com>

8. Voir annexe 2.

9. Texte intégral ici : < http://parlemonde.mascenenationale-creative.com/ index.php/2016/12/06/cest-a-dire-texte-de-frederic-dumond/>.

10. Conférence DGSCO, 2 mai 2017, non publiée.

11. Winnicott (1971).

12. Élève péruvien arrivé une semaine avant la résidence de Frédéric Dumond.

13. Actuation est ici un hispanisme, « actuación » signifiant en espagnol le jeu (au sens théâtral).

14. Nous rappelons que ce n'était pas la consigne dans le cas présent, mais c'est l'habitus scolaire de J.

15. Expressions de Frédéric Dumond.

16. Interview accordée au Boston Society Globe le $1^{\mathrm{er}}$ août 1971, p. 61.

17. Interview par Thomas Flagel pour le magazine des professionnels du spectacle La Scène, $\mathrm{n}^{\circ} 85$, juin-juillet-août 2017, p. 137.

\section{RÉSUMÉS}

Nous proposons de relier le geste artistique et la compétence langagière en analysant l'expérience du travail mené dans une unité pédagogique pour élèves allophones arrivants par une vingtaine d'élèves allophones scolarisés dans un lycée avec l'artiste, écrivain et perfomer Frédéric Dumond dans le cadre de l'évènement Parlemonde organisé par une scène nationale en collaboration avec un CASNAV. Nous montrerons en quoi un projet artistique d'ampleur, qui s'appuie sur le plurilinguisme d'un territoire et qui est fondé par la notion de rencontre, peut être le catalyseur de l'apprentissage d'une nouvelle langue pour les participants et source de nouvelles dynamiques tant pédagogiques et didactiques que sociales pour tous.

We propose linking art and linguistic skill by analyzing the work performed by a high school class comprised of twenty migrant allophone students with Frédéric Dumond, artist, writer and performer. This work took place within the framework of the event Parlemonde, organized by a national theatre in collaboration with the competent schooling authority. We will demonstrate how a significant artistic project, based on linguistic diversity within a territory, and grounded on the idea of sharing, does act as a catalyst for students to learn a new schooling language and be source of powerful social dynamics for everyone. 
INDEX

Keywords : art project, language learning, allophone student, linguistic skills, plurilingualism, artist, social dynamics, mediation, commitment

Mots-clés : projet artistique et culturel, élève allophone, UPE2A, compétence langagière, plurilinguisme, médiation, artiste, engagement

\section{AUTEURS}

\section{MAUD SÉRUSCLAT-NATALE}

Coordonnatrice CASNAV, doctorante à l'université Paul Valéry Montpellier 3, ED 58, Praxiling

\section{MARYSE ADAM-MAILLET}

Inspectrice d'académie, inspectrice pédagogique régionale de lettres, responsable du CASNAV 